University of New Hampshire

University of New Hampshire Scholars' Repository

Life Sciences Faculty Scholarship

Life Sciences

$1-1-2012$

\title{
Patterns of anxiety symptoms in toddlers and preschool-age children: Evidence of early differentiation
}

\author{
Nicholas D. Mian \\ University of Massachusetts Boston, Nicholas.Mian@unh.edu \\ Leandra Godoy \\ University of Massachusetts Boston \\ Margaret J. Briggs-Gowan \\ University of Connecticut Health Center \\ Alice S. Carter \\ University of Massachusetts Boston
}

Follow this and additional works at: https://scholars.unh.edu/unhmbiology_facpub Comments

This is an Author's Original/Accepted Manuscript of an article published by Elsevier in Journal of Anxiety Disorders in 2012, available online: https://dx.doi.org/10.1016/j.janxdis.2011.09.006. This manuscript version is made available under the CC-BY-NC-ND 4.0 license http://creativecommons.org/licenses/by-nc-nd/4.0/

\section{Recommended Citation}

Mian, N. D., Godoy, L., Briggs-Gowan, M. J. \& Carter, A. S. (2012). Patterns of anxiety symptoms in toddlers and preschool-age children: Evidence of early differentiation. Journal of Anxiety Disorders, 26(1), 102-110. Doi: 10.1016/j.janxdis.2011.09.006

This Article is brought to you for free and open access by the Life Sciences at University of New Hampshire Scholars' Repository. It has been accepted for inclusion in Life Sciences Faculty Scholarship by an authorized administrator of University of New Hampshire Scholars' Repository. For more information, please contact Scholarly.Communication@unh.edu. 


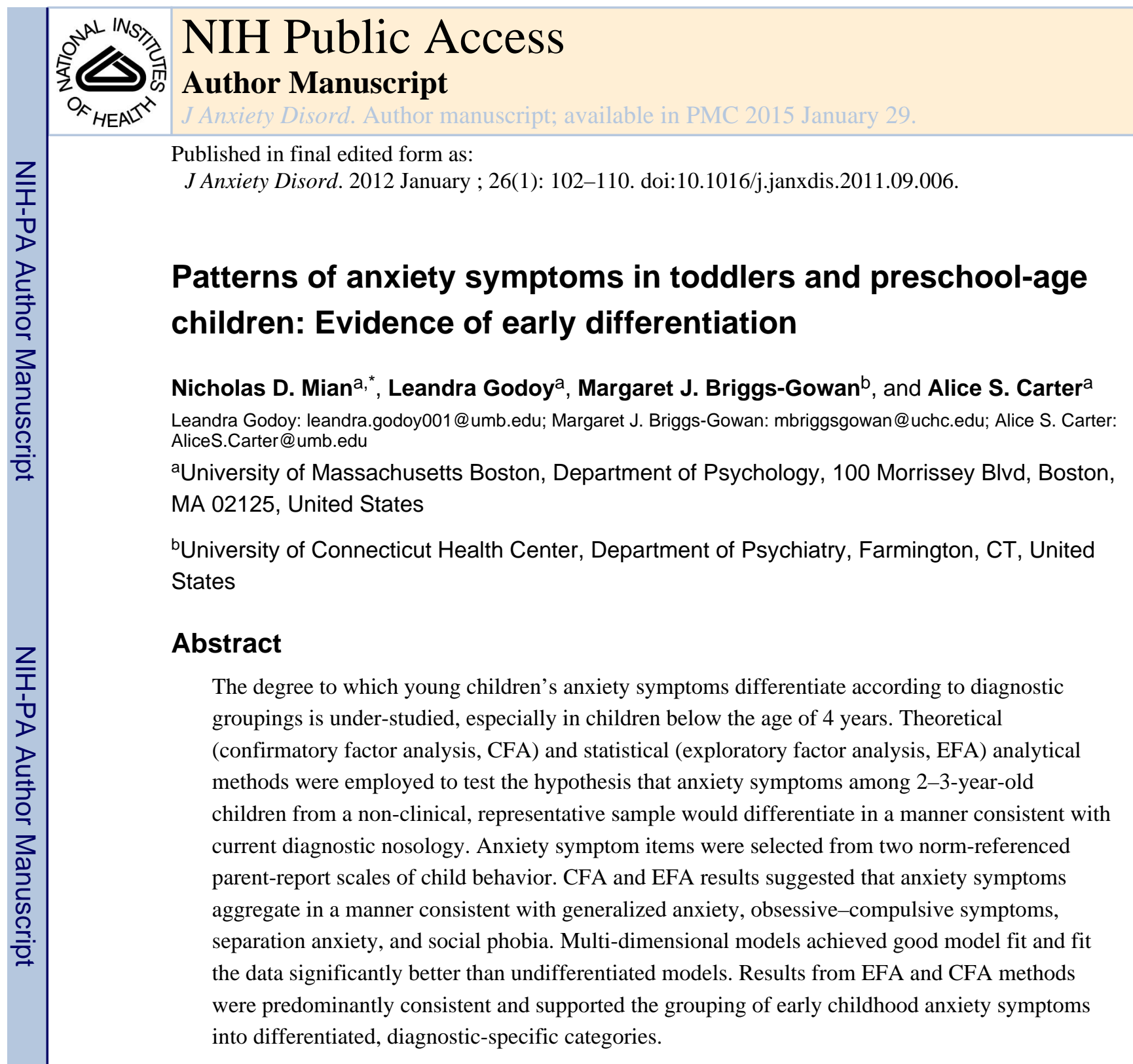

Keywords

Anxiety; Preschool; Nosology; DSM; Factor analysis; Internalizing

\section{Introduction}

Anxiety disorders are among the most frequently occurring childhood psychiatric disorders with prevalence estimates approaching 10\% among preschool children (Egger \& Angold, 2006). Advances in early childhood assessment, albeit still largely dependent on parental reports, now make it feasible to study anxiety symptoms in young children ${ }^{1}$ (Carter, BriggsGowan, \& Davis, 2004; Carter, Godoy, et al., 2010), and evidence of anxiety symptoms is present in children as young as 1 and 2 years of age (Carter, Briggs-Gowan, Jones, \& Little,

\footnotetext{
(C) 2011 Elsevier Ltd. All rights reserved.

"Corresponding author. Tel.: +1 617794 9515. nicholas.mian001@umb.edu (N.D. Mian).

${ }^{1}$ The terms "young children" and "preschool-age children" are used interchangeably to refer to children below age 5 years. We also use the term "early childhood" to refer to children in this age period.
} 
2003; Egger \& Angold, 2006). Supporting the need to be sensitive to development, one study of preschool children (Spence, Rapee, McDonald, \& Ingram, 2001) found that 3-yearolds were significantly more anxious than 4- or 5-year-olds. Moreover, early emerging elevations in anxiety and general internalizing symptoms persist within the preschool period (Briggs-Gowan, Carter, Bosson-Heenan, Guyer, \& Horwitz, 2006) and into the elementary school years (Mian, Wainwright, Briggs-Gowan, \& Carter, 2011). Among school-aged children, anxiety symptoms are not only associated with later anxiety disorders, but also with depression and externalizing disorders in adolescence (Bittner et al., 2007).

Yet, despite interest in examining differentiation in manifestations of anxiety across disorders in older children (Birmaher, Khetarpal, Brent, \& Cully, 1997), little attention has been paid to empirically testing the manner in which early emerging anxiety symptoms aggregate or conform to clinical syndromes that align with diagnostic categories. Such research is needed to elucidate the developmental etiology of anxious emotion and to inform developmentally sensitive clinical taxonomy in children of all ages, from early childhood through adolescence. In the present study, these issues were examined in a representative sample of 2- and 3-year-old children by conducting factor analyses on parent-reported anxiety symptoms from two norm-referenced, developmentally appropriate measures.

\subsection{Background}

The process of revising the Diagnostic and Statistical Manual of Mental Disorders (DSMIV) (American Psychiatric Association, 2000) has highlighted the challenges of improving diagnostic conceptualization and incorporating elements of dimensional and categorical systems relevant to both research and clinical practice (Brown \& Barlow, 2005). An important aspect of this process is improving conceptualizations of developmental phenotypes to address how emotional syndromes and disorders reflect shared and distinct developmental pathways. Factor analytic research in school-age children suggests that the symptoms of different anxiety disorders represent diagnostically distinct groups of symptoms (Spence, 1997). However, it is unclear whether different anxiety disorders emerge as distinct entities or if anxiety disorders begin in an undifferentiated form and become increasingly distinct over time.

In a review of developmental trajectories of anxiety symptoms, Weems (2008) differentiates between primary and secondary features of anxious emotion. Primary features represent the underlying dysregulation of the emotional response system that likely accounts for general risk for anxiety disorder, including physiological (e.g., racing heart), cognitive (e.g., catastrophizing), and behavioral (e.g., avoidance) symptoms. Secondary features represent symptoms that are disorder-specific, such as fear of embarrassment in social anxiety disorder (SAD) or excessive worry in general anxiety disorder. Regrettably, Weems's model does not acknowledge developmental processes before age six, although it offers a theory that may be applicable to younger children. Accordingly, primary features may be relatively stable across development, while secondary features may be less stable, such that children with chronic dysregulation of the anxiety response system may meet criteria for different diagnoses at different stages of development (Costello, Egger, \& Angold, 2004). Thus, it might follow that young children's symptom profiles reflect more general, primary features 
while older children may exhibit more secondary features, suggesting that anxiety symptoms become increasingly differentiated and disorder-specific over time. Despite a lack of evidence for anxiety as a uni-dimensional construct, research in young children often applies a highly undifferentiated approach, lumping all anxiety symptoms together, often with depressive symptoms, as "internalizing symptoms" (Bongers, Koot, van der Ende, \& Verhulst, 2003; Sterba, Prinstein, \& Cox, 2007).

On the other hand, some researchers propose that the diagnostic specificity found in older child and adult anxiety can also be found in young children (Egger \& Angold, 2004; Warren, Umylny, Aron, \& Simmens, 2006), which suggests that even young children experience anxiety in a differentiated manner that may correspond with DSM-IV categories. If supported, this would demonstrate that secondary features (Weems, 2008) of anxiety disorders are present in young children, and may develop concurrently with more general, primary features. A differentiated diagnostic approach would also encourage the more refined study of genetic and ecological risk factors for narrower sets of disorder-specific symptoms (e.g., Hallett, Ronald, Rijsdijk, \& Eley, 2009; Rapee \& Spence, 2004). Although most empirical work on treatment efficacy for older children still lumps anxiety disorders (with the exception of panic disorder and obsessive compulsive disorder [OCD]), differentiating between diagnostic profiles has been integral to the development of empirically supported treatments for specific problems (i.e., Kendall, 2006). From a developmental perspective, one question then is at what point in development should anxiety be treated as differentiated?

\subsection{Anxiety symptom differentiation in children}

Research documents differentiation in early anxiety and depressive symptom presentations (Briggs-Gowan \& Carter, 1998; Carter et al., 2003; Egger, Ascher, \& Angold, 1999) and patterns of risk (Marakovitz, Wagmiller, Mian, \& Carter, 2011). Moreover, varied developmental and gender patterns are observed in young child trajectories of internalizing subscales that are masked when symptoms are viewed as an undifferentiated internalizing construct (Carter, Godoy, et al., 2010). However, few studies examine models of differentiation of internalizing symptoms among children below the age of 5, and especially below the age of 3 (Egger \& Angold, 2006). Assessment challenges may contribute to the paucity of research on anxiety symptoms in very young children; yet prior research has demonstrated that parents are able to reliably identify and categorize their children's problem behaviors, including symptoms of anxiety (Achenbach \& Rescorla, 2000; Carter, Little, Briggs-Gowan, \& Kogan, 1999; Carter et al., 2003). Given the limited research on clustering of anxiety symptoms in preschool- and toddler-age children, we review studies of anxiety symptoms among school-age children. There is substantial heterogeneity across studies regarding methods (e.g., items used, child vs. parent informant), samples (e.g., clinical vs. convenience), and analytical approaches used. However, research on the covariation of symptoms typically uses factor analysis, which is an ideal statistical approach for the task because it identifies the ways individual symptoms relate to one another or "hang together," uncovering the latent groupings of symptoms that may represent meaningful clinical presentations. Results from relevant studies reviewed are summarized in Table 1. 
Studies with school- and preschool-aged children have provided evidence of differentiation that closely maps onto DSM-IV categories despite employing wide age ranges, different item sets, and both clinical and non-clinical samples (see Table 1). The number of factors found among anxiety-related symptoms has ranged from three to six. Factors for symptoms related to separation anxiety disorder (SAD) and social phobia (SP) have been found consistently in reviewed studies that have included relevant items. OCD-related symptoms have also emerged relatively consistently, but to a lesser degree. The degree to which symptoms related to generalized anxiety disorder (GAD) are consistently found within a single factor is unclear, since these symptoms have been investigated as (1) a GAD syndrome, (2) a GAD/depression syndrome, or (3) a related syndrome comprising "general distress" or "physical symptoms". The only study that found evidence for a uni-dimensional model resulted in poor model fit (Muris, Merckelbach, Schmidt, \& Mayer, 1999).

A detailed investigation of studies with preschool children suggests that differentiation of anxiety symptoms according to diagnostic categories can be found in young children. Spence et al. (2001) found that a five-factor model, including SP, SAD, OCD, fears of physical injury and GAD, provided a good fit for the data and was superior to a one-factor model representing undifferentiated symptoms. Although symptoms related to GAD and SAD represented distinct factors, a high correlation between them $(r=0.87)$ suggested a significant association. Sterba, Egger, and Angold (2007) and Sterba, Prinstein, et al. (2007) used items from the Preschool Age Psychiatric Assessment (Egger \& Angold, 2004), a semistructured diagnostic parent interview, and found that a three-factor model including SAD, SP and combined generalized anxiety/depression fit the data better than either a unidimensional model or a more differentiated model of anxiety symptoms (Sterba, Egger, et al., 2007). In general, results of these studies suggest that use of assessment tools that capture a range of diagnostic presentations enables the identification of differentiated subtypes of anxiety in preschoolers. Finally, Eley et al. (2003) examined anxiety differentiation in a large, population-based sample of 4-year-old twin pairs whose mothers completed a short (16-item) checklist. The authors found that a five-factor model of anxiety, including general distress, SAD, fears, obsessive-compulsive behaviors, and shyness/ inhibition was the best fit for the data (Eley et al., 2003). Thus, the limited research among preschool-aged children supports the notion that early childhood anxiety symptoms may cluster into diagnostic-specific groupings, similar to those found among older children.

A major limitation of the current literature is the considerable variation across studies regarding how anxiety symptoms are conceptualized and measured, leading to challenges in interpreting and comparing findings. Most studies have investigated the factor structure of anxiety symptoms in children in the context of symptom measure validation. Hence, only one measure is used in the analysis, and what is tested is how well the data fit the item construction of this particular measure. Because different measures have been designed to assess specific dimensions of anxiety and may not cover all related disorders, different factor profiles may reflect differences in domain coverage. Aggregating more general measures of child behavior that were not specifically designed to test differentiation according to diagnostic profiles may help mitigate the influence of a particular research group's conceptualization. This approach also allows for beginning analyses with a large 
pool of previously validated items. Variation across studies also reflects inconsistency regarding which disorders are included in analyses. Some studies investigate anxiety symptoms alone while others include depressive symptoms; others investigate anxiety "behaviors" rather than symptoms. Investigations of the broad internalizing domain are important, but focusing on anxiety symptoms exclusively provides a more precise picture of early anxiety. Another limitation is that most studies have employed either clinical or convenience samples, which may not reflect the way in which anxiety symptoms differentiate for children not represented. Since we know that a large number of young children with social-emotional problems do not receive clinical services (Kataoka, Zhang, \& Wells, 2002), it is important to expand research to include epidemiological, or representative, community samples. Finally, further investigations with younger children are necessary to elucidate the manner in which latent symptom structures reflect developmental trajectories of anxious emotion (Weems, 2008).

\subsection{The present study}

The present investigation employed confirmatory factor analysis to investigate the underlying structure of anxiety symptoms among children $2-3$-years-old as measured by anxiety items selected from two parent-report scales of child behavior. Following confirmatory modeling we sought to determine if findings would be supported by exploratory factor analysis. This analytic approach has been recommended for studies of latent symptom patterns (Brown \& Barlow, 2005); it provides a way to "check" that findings are statistically sound while also prioritizing theory-driven hypothesis testing. The following hypotheses were made: (1) parent reports of selected child behaviors/symptoms will load onto four correlated factors that represent diagnostic categories: GAD, SAD, SP, and OCD; (2) a multi-dimensional model will explain observed patterns better than a uni-dimensional or undifferentiated model in which all symptoms load on a single factor; (3) theoretically derived, confirmatory models that impose relations between items and diagnostic categories will be corroborated by statistical methods that derive relations between items and latent categories (i.e., exploratory factor analysis).

\section{Method}

\subsection{Participants}

The current work represents secondary analysis of data collected within a longitudinal study of an age- and sex-stratified random population sample, initially ascertained from birth records provided by the State of Connecticut for children born from July 1995 to September 1997 at Yale New Haven Hospital (for greater detail, see Carter, Wagmiller, et al., 2010).

Based on birth record data, children at risk for developmental delays because of prematurity, low birth weight, low APGAR scores, birth complications, or long newborn hospital stay or those with an already-sampled sibling were excluded $(n=971 ; 12 \%)$. After applying exclusions, 7433 families were eligible and, based on power analysis and feasibility, 1788 were randomly sampled. Families were excluded if they had moved out of the state, the child was not in the custody of a biological parent, or if neither parent spoke English ( $n=$ 183). Additionally, children were excluded if they could not be located despite intensive search efforts $(n=112)$ and in two cases, due to severe parent illness. Of 1491 eligible 
families, $86 \%(n=1280)$ participated in Year 1. These participants were similar in sociodemographic characteristics to families living in the New Haven-Meriden Standard Metropolitan Statistical Area of the 1990 Census (Carter, Wagmiller, et al., 2010).

In the present study, data from Year 2 were examined due to the larger portion of parents who were able to complete the CBCL/2-3, which was completed only by parents of children 18 months and older. The Year 2 sample $(n=1219)$ included 49 families that were selected but participated for the first time in Year 2, reflecting 91.4\% retention and an $81.8 \%$ overall response rate. Seventeen families were deemed ineligible due to developmental delays and genetic disorders (e.g., autism spectrum, Fragile X). In this report, only data completed by birth mothers $(n=1110)$ are included. Children included in present analyses ranged from 22.6 to 47.9 months $(M=36.06, S D=6.84)$. The sample was diverse with respect to key sociodemographic characteristics. The sample was approximately equally distributed in terms of child sex ( $47.7 \%$ boys). With regard to race and ethnicity, parents identified their children as being white (65.9\%), African American/Black (17.5\%), Hispanic (5.1\%), Asian $(1.8 \%)$, multi-racial/ethnic including black or Hispanic (8.4\%), and other (1.3\%). Maternal education varied, including $7.6 \%$ who had not completed high school, $17.5 \%$ with a high school degree or equivalent, $33.8 \%$ with some education beyond high school, and $41.2 \%$ with at least a college degree. Approximately $19.7 \%$ of households were single parent homes. Poverty estimates, based on household income and family composition, indicated that $14.8 \%$ of families were living below the federal poverty line and $16.4 \%$ of families were living in borderline poverty (with incomes above $100 \%$ but below $185 \%$ of the poverty line).

\subsection{Procedure}

Parents were invited by mail to complete a survey about their child and family life when their child was between 11.5 and 23 months of age. Those who completed this survey were asked to complete a second survey, used in this report, the following year. Most parents completed the surveys independently; less than one percent was interviewed. Several measures were taken to encourage participation (e.g., sending a children's book with the questionnaire booklet, telephoning, visiting their homes). Parents received $\$ 25$ for each questionnaire they completed. All procedures were approved by two university institutional human subjects review boards. Informed consent was obtained from all participants.

2.2.1. Item selection-Assignment of items to anxiety disorder clusters was determined by consensus by one PhD-level and two Masters-level clinical psychologists and confirmed by one senior, PhD-level researcher (the fourth author). Instances of non-consensus, which were rare, were resolved by consultation with this senior researcher. Items from the Child Behavior Checklist (Achenbach, 1992) and the Infant-Toddler Social and Emotional Assessment (ITSEA; Carter et al., 2003) were reviewed and included if there was consensus that the item assessed one of the following specific dimensions of anxiety: generalized anxiety, separation anxiety, social phobia, and obsessive-compulsive symptoms, corresponding to GAD, SAD, SP, and OCD. Items that were judged to assess non-anxiety symptoms (e.g., hits, kicks, bites) or general behaviors that may reflect a variety of problems (e.g., cries a lot, looks unhappy) were excluded from consideration. It was determined that although anxiety in young children can manifest as other emotional and/or disruptive 
behaviors, including these items would add substantial ambiguity to model interpretation. In addition, due to sampling in a young, non-clinical sample, certain diagnostic categories, such as panic disorder and post-traumatic stress disorder, were not investigated. Finally, items relating to specific phobias were not included because they related specifically to various, distinct fears and, therefore, were not appropriate for factor analysis.

\subsection{Measures}

2.3.1. Sociodemographic information-Parent questionnaire responses and birth records were used to obtain family sociodemographic data (e.g., ethnicity, poverty status, parental education, and marital status). Poverty was defined by federal income guidelines and calculated using reported income and family composition (number of children and adults living in the home).

2.3.2. Infant-Toddler Social and Emotional Assessment (ITSEA)—The ITSEA, a 166-item parent report questionnaire, assesses four broad domains of behaviors (Externalizing, Internalizing, Dysregulation, and Competencies) as well as low base rate social problem indices (Maladaptive, Atypical Behavior, and Social Relatedness) in 12-48month olds (Carter \& Briggs-Gowan, 2006). Items are rated on the following 3-point scale: (0) Not true/rarely, (1) Somewhat true/sometimes, and (2) Very trueloften. A "No opportunity" code allows parents to indicate that they have not had the opportunity to observe certain behaviors (e.g., behavior with peers). Across several studies, the ITSEA has demonstrated acceptable internal consistency ( 0.85 for the internalizing domain), 6-day testretest reliability ( $r=0.85$ for the internalizing domain), as well as validity relative to other parent report checklists and independent behavioral observations (Carter \& Briggs-Gowan, 2006; Carter et al., 2003). The analyses for this paper include four items that were used in the original development of the ITSEA, but are not included in the final version. Items presented have been abbreviated due to copyright restrictions.

2.3.3. Child Behavior Checklist 2-3 (CBCL/2-3)-The CBCL/2-3, a 99-item parent report questionnaire, assesses problem behavior in 2- and 3-year-olds (Achenbach, 1992) was completed by parents in this study. The measure consists of Internalizing, Externalizing, and Total Problem domains. It is very similar to a slightly revised and re-normed version of the CBCL, the CBCL/1.5-5, (Achenbach \& Rescorla, 2000) which is appropriate for use with 18-60-month-old children. The two versions differ minimally: of the 99 items on the CBCL/2-3, only two were replaced and 6 were re-worded slightly. Information from the CBCL/2-3 can be used to derive scores on the CBCL/1.5-5 (e.g., Konold, Hamre, \& Pianta, 2003), and the author of the CBCL granted permission to use the CBCL/2-3 in a manner consistent with the CBCL/1.5-5 (i.e., with children from 18 months to 5 years). The measure has demonstrated very good 8-day test-retest reliability $(r=0.68-0.92$, mean $r=$ 0.84 ), cross-informant agreement (mean mother-father $r=0.61$, mean parent-child care $r=$ 0.65 ), and success in discriminating between referred and non-referred children. Items in tables have been abbreviated. 


\subsection{Data analytic plan}

Data were analyzed with two statistical packages: SPSS Version 16 was used for descriptive statistics and correlation analyses, and Mplus Version 5.1(Muthen \& Muthen, 2007) was used for exploratory factor analysis (EFA) and confirmatory factor analysis (CFA). Four fit indices were used to evaluate factor analyses: (1) the Bentler Comparative Fit Index (CFI) ranges from 0.0 to 1.0; this study used a cut-off value of 0.9 to represent adequate fit and 0.95 representing "good fit" (Hu \& Bentler, 1999). (2) The root mean square error of approximation (RMSEA) is minimally sensitive to sample size; values less than 0.05 are generally considered a "very good fit," while values less than 0.1 represent "reasonable fit" (Fan, Thompson, \& Wang, 1999). (3) The standardized root mean square residual (SRMR) ranges from 0 to 1.0; values less than 0.08 considered adequate (Hu \& Bentler, 1999). (4) The Chi square $\left(\chi^{2}\right)$ statistic is reported but not used as a measure of fit, as it is a poor estimate with large samples (Fan et al., 1999). The $\chi^{2}$ statistic was used to compare differences in fit between nested models.

Items were selected from the ITSEA and CBCL. CFA was conducted with a maximum likelihood estimator. Mplus uses full information maximum likelihood to account for missing data. Although items included in the CFA were selected using the theoretical approach described above, model development was also informed by modification indices. Then, an EFA was conducted with the same items to examine consistency between methods. To investigate age effects, the sample was divided into two age groups using a median split. Multi-group analyses using an equality-constraints method (Kline, 1998) were conducted.

\section{Results}

\subsection{Theoretically derived confirmatory factor analysis}

A large pool of 75 items, thought to be potentially related to anxiety symptoms, was considered ( 31 from the CBCL, 44 from the ITSEA). Before conducting CFA, the item pool was narrowed by inter-rater consensus for items deemed the strongest candidates for capturing symptoms related to DSM-IV anxiety disorders. This resulted in a hypothesized model with 36 total items (12 CBCL items and 24 ITSEA items) loading on four factors representing GAD, SAD, SP, and OCD. Correlated errors based on modification indices were allowed only when it was agreed that it was theoretically appropriate for items due to high likelihood of shared method variance (e.g., two items probing the same symptom). This model represented an inadequate fit for the data $\left(\chi^{2}=2074.40, d f=575 ; \mathrm{CFI}=0.839\right.$; RMSEA $=0.048,90 \% \mathrm{CI}=0.046-0.051$; SRMR $=0.053$; fit indices for all CFA models are presented in Table 4). Thus, two items with low factor loadings (restless, can't sit still; wants to sleep in someone else's room or bed) were trimmed from the model, resulting in a model (presented in Table 2) with 34 items (12 CBCL items and 22 ITSEA items). The same correlated errors were allowed in this model. For all factors, indicators loaded significantly $(p<0.001)$ with standardized loadings ranging from 0.277 to 0.733 (only two loadings were below 0.30). All factors were significantly intercorrelated (see Table 3). SAD and SP were most strongly correlated $(r=0.53, p<0.001)$. This model represented a good fit for the data for most fit indices $\left(\chi^{2}=1406.53, d f=508\right.$; CFI $=0.896$; RMSEA $=0.039$, $90 \% \mathrm{CI}=0.037-0.042$; SRMR $=0.048$ ). Only the CFI was slightly below the 0.90 threshold 
for adequate fit. This fit was also significantly better than the 36-item model $\left(\chi^{2} \Delta=667.9\right.$, df $\Delta=67, p<0.001)$.

To test the second hypothesis, that the multi-dimensional model presented above would fit the data better than a uni-dimensional model, the same 34 items were included in a model in which all items loaded on a single, undifferentiated factor. The pairs of correlated errors from the four-factor model were maintained. The fit of this undifferentiated model was poor $\left(\chi^{2}=3384.04, d f=514 ; \mathrm{CFI}=0.667\right.$; RMSEA $=0.071,90 \% \mathrm{CI}=0.069-0.073 ;$ SRMR $=$ $0.079)$, and was significantly worse than the 4-factor model $\left(\chi^{2} \Delta=1977.5, d f \Delta=6, p<\right.$ 0.001 ), indicating that the multi-dimensional, theoretically derived model was a better fit for the data.

\subsection{Exploratory factor analysis}

An EFA was run with oblique rotation (allowing factors to be intercorrelated) with the final pool of 34 items. Results from EFA revealed 9 factors with eigenvalues greater than 1.0 (Cattell, 1966). Items were found to correspond with DSM-IV symptoms of various anxiety disorders, but some disorders were represented as multiple factors, each reflecting different clusters of symptoms. One factor corresponded to SP (5 items); three factors corresponded to GAD, representing "somatic" (3 items), "worry" (4 items), and "concentration" ( 2 items) symptoms; two factors corresponded with OCD, representing "perfection" (6 items) and "repetition" (4 items) symptoms; three factors corresponded with SAD, representing "separation" (4 items), "clinginess" (4 items), and sleep-related concerns (2 items). EFA loadings for these items are presented in Table 2, along with corresponding CFA loadings. All items except two were above 3.0 in EFA. All items except two clearly loaded on one factor, as evidenced by a significantly elevated factor loading (above 0.30 ) on only one factor. The item, "gets too upset when separated from parents," loaded on two factors relating to SAD (separation: 0.402 and clinginess: 0.310 ) while the item, "puts things in a special order over and over," loaded on both factors relating to OCD (perfection: 0.37 and repetition: 0.415 ). Although many items loaded on factors that represented smaller groups of symptoms within the hypothesized factor, all items loaded on factors that were consistent with the 4-factor theoretical model. As can be seen in Table 3, the 9 factors derived from the EFA were significantly intercorrelated with the exception of factors relating to SP and concentration. GAD factors relating to somatic and worry symptoms were highly correlated $(r=0.701)$, as were the factors relating to separation and clinginess $(r=0.780)$, both relating to SAD. The sleep-related factor loaded most highly on separation and clinginess, suggesting that sleep-related symptoms are most related to SAD. Of note, both factors relating to OCD, repetition and perfection, were also highly correlated $(r=0.695)$.

\subsection{Investigation of age effects}

Two models for children older and younger than the median age (36.3 months) were run using the items and factor configuration from the final CFA model described above. Both models (each $n=555$; mean ages $=30.2$ and 42.0 months) represented an acceptable fit to the data, with almost identical fit indices $\left(\chi^{2}=1075, d f=508\right.$; CFI $=0.873$; RMSEA $=$ 0.045 ; SRMR $=0.056$ and 0.054 for the younger and older groups, respectively), suggesting that the configuration of factors was upheld for both age groups. Testing individual factor 
loadings for invariance by comparing constrained and unconstrained models revealed that three CBCL items, all on the GAD factor, varied by age: worrying (standardized loadings were 0.69 vs. 0.46 for younger and older children, respectively), can't concentrate pay attention for long (0.21 vs. 0.54$)$, and can't sit still/restless/hyperactive (0.23 vs. 0.51$)$. All loadings were significant at $p<0.001$.

\section{Discussion}

The purpose of this study was to investigate whether anxiety symptoms present in 2-3-yearold children from a representative sample cluster in a manner consistent with current diagnostic nosology. The hypothesis that symptoms would differentiate in a manner consistent with current diagnostic DSM-IV nosology was generally supported. Anxiety symptoms aggregated in a manner consistent with current understanding of GAD, OCD, $\mathrm{SAD}$, and SP. The differentiated model performed better than the undifferentiated (unidimensional) model across fit indices. Moreover, the undifferentiated model failed to achieve adequate fit. Results achieved by EFA corroborated theoretically driven CFA analyses, thus providing further evidence that items in this sample were best represented as differentiated factors. Finally, the configuration of the four hypothesized factors was largely supported for both younger and older children.

Although this study provided general support for a four-factor model with groups of symptoms representing GAD, OCD, SAD, and SP, there was some inconsistency across findings derived from CFA and EFA methods. Of note, EFA produced a factor structure in which symptoms were represented by nine factors, rather than the four theorized factors. Upon examination, these nine factors appear to represent "sub-factors" or clusters of symptoms that capture different aspects of the four diagnostic syndromes. Although these results were consistent with our hypothesis that symptoms would be best represented as various factors rather than one undifferentiated factor, we did not predict that symptoms would differentiate further than has been found in older children.

Three of the diagnostic groupings were comprised of multiple factors according to EFA methods. In the case of obsessive-compulsive symptoms, this is not surprising, as much research has suggested that OCD is a disorder characterized by heterogeneity (Bloch, Landeros-Weisenberger, Rosario, Pittenger, \& Leckman, 2008). Although this two-factor representation of OCD symptoms representing "perfectionism" and "repetition" is not entirely consistent with the four-factor solution (symmetry, forbidden thoughts, cleaning and hoarding) often found in clinical samples of adults and older children (Bloch et al., 2008), it is consistent with work with young (8-72 months old) children (Evans, Leckman, Carter, \& Reznick, 1997) and suggests that OCD presents as a complex set of symptoms even in young children.

The finding that GAD symptoms loaded on three factors representing "worry," "somatic," and "concentration" symptoms is somewhat consistent with research with older children using diagnostic measures (Higa-McMillan, Smith, Chorpita, \& Hayashi, 2008). However, three items on the GAD factor varied by age. Both items comprising the "concentration" factor were more important (had larger loadings) for older children, reflecting symptoms 
that may emerge with development, or, in this case, may partially reflect symptoms associated with early emerging attention deficit-hyperactivity disorder. Parents are probably less likely to notice that very young children have trouble concentrating, perhaps due to different expectations for older versus younger children. Worrying, on the other hand, was more important for younger children. This finding is more difficult to interpret-it may be that worrying itself becomes more differentiated over time and is captured by other items, such as concentration difficulties, in older children. In general, these findings suggest that GAD symptoms, compared to other anxiety symptoms, may be less stable at this point in development.

Separation anxiety was also represented as three factors in the EFA- "clinginess," "separation," and "sleep" symptoms. In contrast, March, Parker, Sullivan, and Stallings (1997) investigation of school-age children suggested that separation anxiety was the only subscale of the Multidimensional Anxiety Scale for Children that did not subdivide into multiple factors. This difference may be attributed to age differences; older children are more likely to have problems with separating and independence from caregivers as opposed to the physical closeness represented in the "clinginess" factor found here with toddlers (e.g., "wants to be on lap"). Items related to sleep problems were originally included due to the high overlap between sleep problems and anxiety symptoms in older children (Alfano, Ginsburg, \& Kingery, 2007). These items were considered by authors to be relevant to most anxiety disorders and particularly to SAD. However, the finding that they loaded on their own factor lends support for the distinctiveness of clinically relevant syndromes for sleep problems (Goodlin-Jones \& Anders, 2004). It is possible that sleep-related anxiety symptoms were highly correlated with each other because they most often occur in the same context - at home, often at bedtime or during the night. The regular context of bedtime might lead parents to respond to sleep-related questions consistently, while their responses to non-sleep related anxiety questions might be more variable. However, it is important to note that this factor was most highly correlated with the other SAD factors, suggesting that sleep-related problems are more relevant to SAD than other disorders.

Although symptoms in EFA loaded on a higher number of sub-factors than expected, they did not load on different factors than were hypothesized. Hence, results suggest that symptoms in this age group do indeed differentiate into meaningful clinical syndromes. In general, if the representation of GAD, SAD, and OCD as multiple sub-factors is replicated in other samples, it would suggest the need for developmental modification of the categories that are applied to older children to reflect the manner in which early anxiety symptoms manifest in young children. However, results do suggest that a four-factor model, as is used in older children, is a more appropriate approach than treating anxiety symptoms as undifferentiated in this age group. The incorporation of dimensions in future systems of nosology may clarify how these diagnostic presentations change over the course of development, as such approaches allow for within-category heterogeneity and inform etiological theory and treatment development.

A major challenge in studying anxiety symptom differentiation is the notable inconsistency of methods and findings across priori studies, complicating aggregation of results. However, most studies with school-age children provide evidence of symptom differentiation (see 
Section 1.1). Moreover, the available studies with preschool-age samples found results that are generally similar to those in the present study, identifying either three (Sterba, Egger, et al., 2007) or five factors (Eley et al., 2003; Spence et al., 2001). The hypothesized model supported by the present study is very similar to the two studies providing evidence of five factors. The factors found by Spence et al. (2001) closely corresponded with the factors in the present study - generalized anxiety, obsessive-compulsive symptoms, separation anxiety, and social anxiety - with the exception of their "fears of physical injury" factor, which was not included in the present investigation due to a lack of appropriate items. The five factors found by Eley et al. (2003) were also consistent with the present results. Two factors, separation anxiety and obsessive-compulsive symptoms, were parallel with our findings. The shyness/inhibition factor they found overlapped with social phobia since two of the three items related specifically to social situations. Their "general distress" factor overlapped with our general anxiety factor; one (of four) items represented worry and one represented somatic complaints (although "general distress" also included two depression items).

The evidence of differentiation found in the present sample of very young children has implications for how researchers conceptualize the development of psychopathology. Within a transactional, bioecological framework (Bronfenbrenner \& Ceci, 1994) in which the child and environment have reciprocal influences that develop over time, it might be expected that such transactional processes would first present as a less differentiated, vague symptom or "risk" profile that would become more diagnostically specific as the child develops. Results from multivariate genetic studies have led to related theories of "general genes," which might enhance vulnerability to a variety of clinical problems that become diagnostically specific with environmental influence (Eley, 1997). Findings from the present study suggest that if such processes that narrow the clinical presentation of psychopathology exist, they are already well under way in early childhood. Weems (2008) also suggests that anxiety trajectories reflect primary features that represent general anxiety dysregulation and more specific secondary features reflecting diagnostically distinct syndromes. Again, results from the present study suggest that such secondary features are present even in very young children. Related to this model, the analyses in this report shed light on the ongoing difficulty with distinguishing between symptoms and temperament with young children.

Findings from the present study should be considered in the context of several important limitations. First, all results are from a single, maternal informant. Although this method is appropriate with this age group, who are unable to provide self-reports, inability to assess cognitive, affective, or somatic symptoms from the child's or others' perspective or via observation is a limitation. Second, data included in analyses were cross-sectional, and, although a comparison of two age groups was conducted, this investigation could not demonstrate longitudinal stability or change of symptom presentations over time. Third, only anxiety symptoms were included in analyses, so we are unable to determine how other internalizing symptoms (e.g., depression/withdrawal) may have affected results. In this age group, symptoms reflecting depression/withdrawal are difficult to assess and investigate; when measured, questionnaire items typically have very low base rates (Carter et al., 2003). Although these items were not included because they were beyond the scope of this 
investigation - hypotheses specifically targeted the differentiation of anxiety symptoms future investigations are needed to clarify the relationship between general anxiety and depression/withdrawal in preschool samples (Sterba, Egger, et al., 2007). Fourth, although this study addressed a gap in previous research by using a representative community sample, it is possible that different patterns might emerge in clinically enriched samples of very young children. Future research may consider replication with a clinical sample, investigating other internalizing symptoms, and using other assessment methods such as observation.

Results from the present study lend further support for the differentiation of anxiety symptoms in young children by extending findings to a sample of 2-3-year-olds. Such evidence has implications for how anxiety symptoms should be measured and presented in research. It is advised that early childhood researchers employ approaches that reflect the nuanced differentiation of internalizing symptom presentations. Moreover, clinicians working with young anxious children can use these findings to inform their approach to careful assessment and intervention.

\section{Acknowledgments}

Support for this research came from a grant to the fourth author from the National Institute of Mental Health (R01MH55278). We also wish to thank Matthew Idzik and Meagan Hilton, as well as all the children and families who participated in this project.

\section{References}

Achenbach, TM. Manual for the Child Behaviour Checklist/2-3 and 1992 profile. Burlington, VT: University of Vermont, Department of Psychiatry; 1992.

Achenbach, TM.; Rescorla, LA. Manual for the ASEBA preschool forms and profiles. Burlington, VT: University of Vermont, Department of Psychiatry; 2000.

Alfano CA, Ginsburg GS, Kingery JN. Sleep-related problems among children and adolescents with anxiety disorders. Journal of the American Academy of Child \& Adolescent Psychiatry. 2007; 46(2):224-232. [PubMed: 17242626]

American Psychiatric Association. Diagnostic and statistical manual of mental disorders. 4. Washington, DC: American Psychiatric Association; 2000. text revision

Birmaher B, Khetarpal S, Brent D, Cully M. The Screen for Child Anxiety Related Emotional Disorders (SCARED): scale construction and psychometric characteristics. Journal of the American Academy of Child \& Adolescent Psychiatry. 1997; 36(4):545-553. [PubMed: 9100430]

Bittner A, Egger HL, Erkanli A, Costello EJ, Foley DL, Angold A. What do childhood anxiety disorders predict? Journal of Child Psychology and Psychiatry. 2007; 48(12):1174-1183. [PubMed: 18093022]

Bloch MH, Landeros-Weisenberger A, Rosario MC, Pittenger C, Leckman JF. Meta-analysis of the symptom structure of obsessive-compulsive disorder. The American Journal of Psychiatry. 2008; 165(12):1532-1542. [PubMed: 18923068]

Bongers IL, Koot HM, van der Ende J, Verhulst FC. The normative development of child and adolescent problem behavior. Journal of Abnormal Psychology. 2003; 112(2):179-192. [PubMed: 12784827]

Briggs-Gowan MJ, Carter AS. Preliminary acceptability and psychometrics of the Infant-Toddler Social and Emotional Assessment (ITSEA): a new adult-report questionnaire. Infant Mental Health Journal. 1998; 19(4):422-445. 
Briggs-Gowan MJ, Carter AS, Bosson-Heenan J, Guyer AE, Horwitz SM. Are infant-toddler socialemotional and behavioral problems transient? Journal of the American Academy of Child \& Adolescent Psychiatry. 2006; 45(7):849-858. [PubMed: 16832322]

Bronfenbrenner U, Ceci SJ. Nature-nuture reconceptualized in developmental perspective: a bioecological model. Psychological Review. 1994; 101(4):568-586. [PubMed: 7984707]

Brown TA, Barlow DH. Dimensional versus categorical classification of mental disorders in the fifth edition of the diagnostic and statistical manual of mental disorders and beyond: comment on the special section. Journal of Abnormal Psychology. 2005; 114(4):551-556. [PubMed: 16351377]

Carter, AS.; Briggs-Gowan, MJ. Infant-Toddler Social and Emotional Assessment (ITSEA). San Antonio, TX: Psychological Corporation Harcourt Press; 2006.

Carter AS, Briggs-Gowan MJ, Davis NO. Assessment of young children's social-emotional development and psychopathology: recent advances and recommendations for practice. Journal of Child Psychology and Psychiatry. 2004; 45(1):109-134. [PubMed: 14959805]

Carter AS, Briggs-Gowan MJ, Jones SM, Little TD. The Infant-Toddler Social and Emotional Assessment (ITSEA): factor structure, reliability, and validity. Journal of Abnormal Child Psychology. 2003; 31(5):495-514. [PubMed: 14561058]

Carter AS, Godoy L, Wagmiller RL, Veliz P, Marakovitz S, Briggs-Gowan MJ. Internalizing trajectories in young boys and girls: the whole is not a simple sum of its parts. Journal of Abnormal Child Psychology. 2010; 38(10):19-31. [PubMed: 19662525]

Carter AS, Little C, Briggs-Gowan MJ, Kogan N. The Infant-Toddler Social and Emotional Assessment (ITSEA): comparing parent ratings to laboratory observations of task mastery, emotion regulation, coping behaviors and attachment status. Infant Mental Health Journal. 1999; 20(4):375-392.

Carter AS, Wagmiller RJ, Gray SAO, McCarthy KJ, Horwitz SM, Briggs-Gowan MJ. Prevalence of DSM-IV disorder in a representative, healthy birth cohort at school entry: sociodemographic risks and social adaptation. Journal of the American Academy of Child \& Adolescent Psychiatry. 2010; 49(7):686-698. [PubMed: 20610138]

Cattell, RB. Handbook of multivariate experimental psychology. Chicago: Rand McNally; 1966.

Costello, EJ.; Egger, HL.; Angold, A. Developmental epidemiology of anxiety disorders. In: Ollendick, TH.; March, JS., editors. Phobic and anxiety disorders in children and adolescents: a clinician's guide to effective psychosocial and pharmacological interventions. New York, NY, US: Oxford University Press; 2004. p. 61-91.

Egger, HL.; Angold, A. The Preschool Age Psychiatric Assessment (PAPA): a structured parent interview for diagnosing psychiatric disorders in preschool children. In: DelCarmen-Wiggins, R.; Carter, A., editors. Handbook of infant, toddler, and preschool mental health assessment. New York, NY, US: Oxford University Press; 2004. p. 223-243.

Egger HL, Angold A. Common emotional and behavioral disorders in preschool children: presentation, nosology and epidemiology. Journal of Child Psychology Psychiatry. 2006; 47(3): 313-337. [PubMed: 16492262]

Egger, HL.; Ascher, BH.; Angold, A. The preschool age psychiatric assessment. 1.1. Durham, NC: Center for Developmental Epidemiology, Duke University Medical Center; 1999.

Eley TC. General genes: a new theme in developmental psychopathology. Current Directions in Psychological Science. 1997; 6(4):90-95.

Eley TC, Bolton D, O'Connor TG, Perrin S, Smith P, Plomin R. A twin study of anxiety-related behaviours in pre-school children. Journal of Child Psychology and Psychiatry. 2003; 44(7):945960. [PubMed: 14531577]

Evans DW, Leckman JF, Carter A, Reznick JS. Ritual, habit, and perfectionism: the prevalence and development of compulsive-like behavior in normal young children. Child Development. 1997; 68(1):58-68.10.2307/1131925 [PubMed: 9084125]

Fan X, Thompson B, Wang L. Effects of sample size, estimation methods, and model specification on structural equation modeling fit indixes. Structural Equation Modeling. 1999; 6(1):56-83.

Goodlin-Jones, BL.; Anders, TF. Sleep disorders. In: DelCarmen-Wiggins, R.; Carter, A., editors. Handbook of infant, toddler, and preschool mental health assessment. New York, NY, US: Oxford University Press; 2004. p. 271-288. 
Hallett V, Ronald A, Rijsdijk F, Eley TC. Phenotypic and genetic differentiation of anxiety-related behaviors in middle childhood. Depression and Anxiety. 2009; 26(4):316-324. [PubMed: 19194998]

Higa-McMillan CK, Smith RL, Chorpita BF, Hayashi K. Common and unique factors associated with DSM-IV-TR internalizing disorders in children. Journal of Abnormal Child Psychology. 2008; 36(8):1279-1288. [PubMed: 18607718]

$\mathrm{Hu}$ L, Bentler PM. Cutoff criteria for fit indexes in covariance structure analysis: conventional criteria versus new alternatives. Structural Equation Modeling. 1999; 6(1):1-55.

Kataoka SH, Zhang L, Wells KB. Unmet need for mental health care among U.S. children: variation by ethnicity and insurance status. The American Journal of Psychiatry. 2002; 159(9):1548-1555. [PubMed: 12202276]

Kendall, PC. Child and adolescent therapy: cognitive-behavioral procedures. 3. New York, NY, US: Guilford Press; 2006.

Kline, RB. Principles and practice of structural equation modeling. New York, NY: The Guilford Press; 1998.

Konold TR, Hamre BK, Pianta RC. Measuring problem behaviors in young children. Behavioral Disorders. 2003; 28(2):111-123.

Marakovitz S, Wagmiller RL, Mian ND, Carter AS. Lost toy? Monsters under the bed? Contributions of temperament and family factors to early internalizing problems in boys and girls. Journal of Clinical Child and Adolescent Psychology. 2011; 40(2):233-244. [PubMed: 21391020]

March JS, Parker JDA, Sullivan K, Stallings P. The Multidimensional Anxiety Scale for Children (MASC): factor structure, reliability, and validity. Journal of the American Academy of Child \& Adolescent Psychiatry. 1997; 36(4):554-565. [PubMed: 9100431]

Mian ND, Wainwright L, Briggs-Gowan MJ, Carter AS. An ecological risk model for early childhood anxiety: the importance of early child symptoms and temperament. Journal of Abnormal Child Psychology. 2011; 39(4):501-512.10.1007/s10802-010-9476-9480 [PubMed: 21153696]

Muris P, Merckelbach H, Schmidt H, Mayer B. The revised version of the Screen for Child Anxiety Related Emotional Disorders (SCARED-R): factor structure in normal children. Personality and Individual Differences. 1999; 26(1):99-112.

Muthen, LK.; Muthen, BO. Mplus user's guide. 5. Los Angeles, CA: Muthen \& Muthen; 2007.

Rapee RM, Spence SH. The etiology of social phobia: empirical evidence and an initial model. Clinical Psychology Review. 2004; 24(7):737-767. [PubMed: 15501555]

Spence SH. Structure of anxiety symptoms among children: a confirmatory factor-analytic study. Journal of Abnormal Psychology. 1997; 106(2):280-297. [PubMed: 9131848]

Spence SH, Rapee R, McDonald C, Ingram M. The structure of anxiety symptoms among preschoolers. Behaviour Research and Therapy. 2001; 39(11):1293-1316. [PubMed: 11686265]

Sterba SK, Egger HL, Angold A. Diagnostic specificity and nonspecificity in the dimensions of preschool psychopathology. Journal of Child Psychology and Psychiatry. 2007; 48(10):10051013. [PubMed: 17915001]

Sterba SK, Prinstein MJ, Cox MJ. Trajectories of internalizing problems across childhood: heterogeneity, external validity, and gender differences. Development and Psychopathology. 2007; 19(2):345-366. [PubMed: 17459174]

Warren SL, Umylny P, Aron E, Simmens SJ. Toddler anxiety disorders: a pilot study. Journal of the American Academy of Child \& Adolescent Psychiatry. 2006; 45(7):859-866. [PubMed: 16832323]

Weems CF. Developmental trajectories of childhood anxiety: identifying continuity and change in anxious emotion. Developmental Review. 2008; 28(4):488-502. 


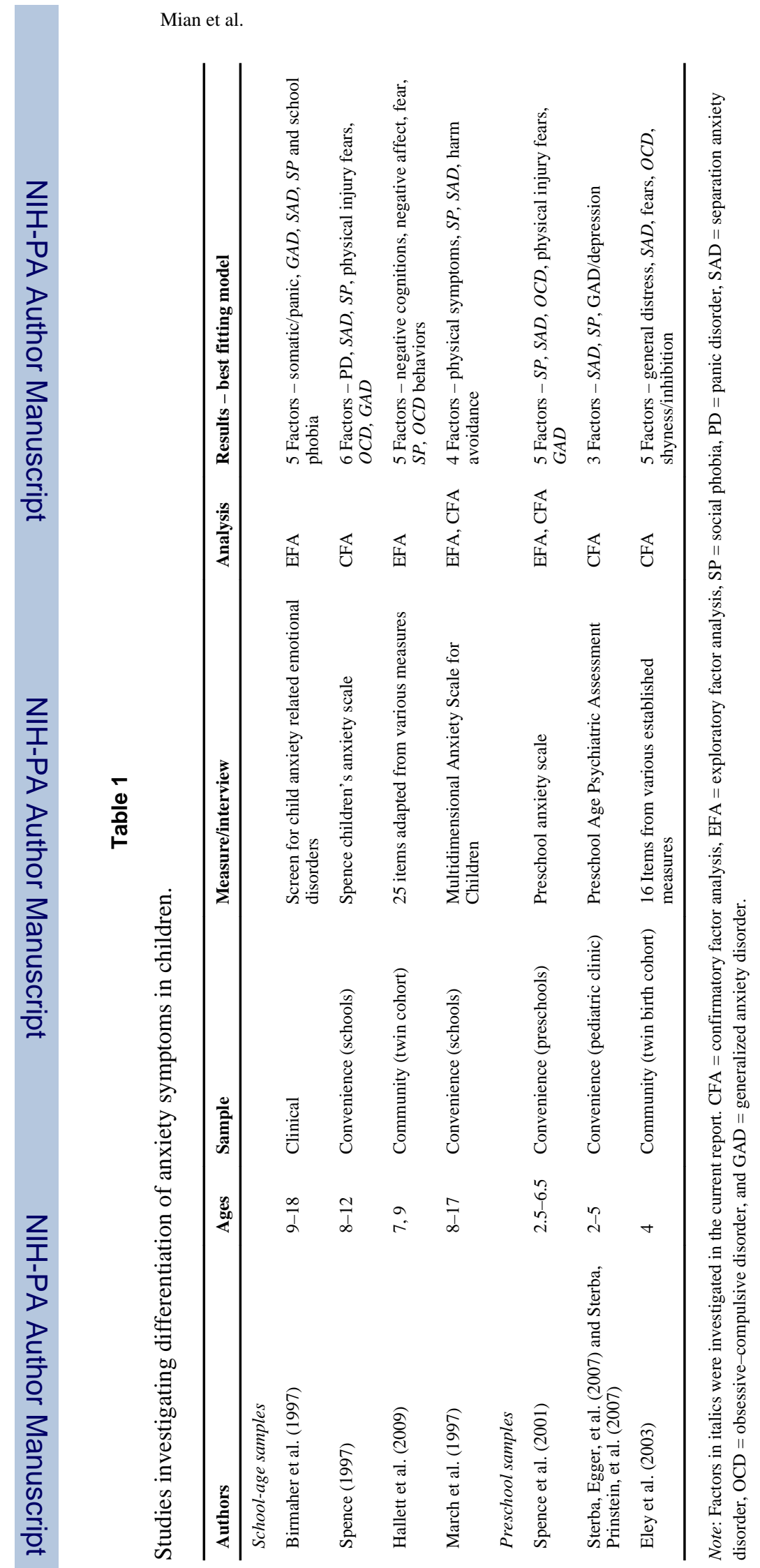

J Anxiety Disord. Author manuscript; available in PMC 2015 January 29. 
Table 2

Items and standardized factor loadings from confirmatory and exploratory factor analysis.

\begin{tabular}{|c|c|c|c|}
\hline \multirow[t]{2}{*}{ CFA factor and items } & \multirow{2}{*}{$\begin{array}{l}\text { CFA } \\
\text { Loading }\end{array}$} & \multicolumn{2}{|l|}{$\underline{\text { EFA }}$} \\
\hline & & Loading & Factor \\
\hline \multicolumn{4}{|l|}{ Social phobia } \\
\hline Shy with groups of new people & 0.69 & 0.80 & SP \\
\hline Shy with new children & 0.66 & 0.58 & SP \\
\hline Shy with new adults & 0.63 & 0.74 & SP \\
\hline While to speak in unfamiliar situations & 0.48 & 0.44 & SP \\
\hline Prefers to play alone/watch others play & 0.37 & 0.27 & SP \\
\hline \multicolumn{4}{|l|}{ Separation anxiety } \\
\hline Clings to adults, too dependent ${ }^{a}$ & 0.64 & 0.60 & Clinginess \\
\hline Hangs on you/wants to be in lap & 0.63 & 0.53 & Clinginess \\
\hline Is very clingy & 0.61 & 0.56 & Clinginess \\
\hline Reaches for you when not holding & 0.40 & 0.30 & Clinginess \\
\hline Gets too upset when separated ${ }^{a}$ & 0.62 & 0.40 & Separation \\
\hline Cries or hangs onto you when try to leave & 0.58 & 0.54 & Separation \\
\hline Upset when left with familiar sitter/relative & 0.41 & 0.72 & Separation \\
\hline Upset when left with new baby-sitter & 0.39 & 0.58 & Separation \\
\hline Does not want to sleep alone ${ }^{a}$ & 0.35 & 0.41 & Sleep \\
\hline Must be held to go to sleep & 0.28 & 0.66 & Sleep \\
\hline \multicolumn{4}{|l|}{ Obsessive-compulsive } \\
\hline Needs things to be clean or neat & 0.73 & 0.75 & Perfectionism \\
\hline Very worried about getting dirty & 0.62 & 0.68 & Perfectionism \\
\hline Too concerned with neatness/cleanliness $a$ & 0.62 & 0.40 & Perfectionism \\
\hline Cannot stand things out of place ${ }^{a}$ & 0.60 & 0.52 & Perfectionism \\
\hline Worries about own body & 0.54 & 0.53 & Perfectionism \\
\hline Perfectionist & 0.45 & 0.60 & Perfectionism \\
\hline Puts things in special order, over and over & 0.57 & 0.42 & Repetition \\
\hline Repeats action or phrase, over and over & 0.34 & 0.77 & Repetition \\
\hline Repeats movement, over and over & 0.33 & 0.59 & Repetition \\
\hline Acts out same pretend theme over and over & 0.30 & 0.32 & Repetition \\
\hline \multicolumn{4}{|l|}{ General anxiety } \\
\hline Worrying ${ }^{a}$ & 0.62 & 0.52 & Worry \\
\hline Nervous, high-strung/tense ${ }^{a}$ & 0.60 & 0.52 & Worry \\
\hline Worries a lot or very serious & 0.46 & 0.61 & Worry \\
\hline Sick when nervous or upset & 0.42 & 0.23 & Worry \\
\hline Nausea, sick (no medical cause) ${ }^{a}$ & 0.46 & 0.67 & Somatic \\
\hline Aches or pains (no medical cause) ${ }^{a}$ & 0.41 & 0.48 & Somatic \\
\hline Stomachaches/cramps (no medical cause) ${ }^{a}$ & 0.38 & 0.69 & Somatic \\
\hline
\end{tabular}




\begin{tabular}{llll}
\hline CFA factor and items & CFA & EFA & \\
\cline { 4 - 4 } & Loading & Loading & Factor \\
\hline Cannot concentrate/pay attention $^{a}$ & 0.31 & 0.61 & Concentration \\
Cannot sit still/restless/hyperactive $^{a}$ & 0.30 & 0.87 & Concentration \\
\hline
\end{tabular}

Note: $p<0.001$ for all loadings. Items in italics varied by age. SP = social phobia.

${ }^{a}$ Child Behavior Checklist item (other items are from the Infant-Toddler Social and Emotional Assessment). 


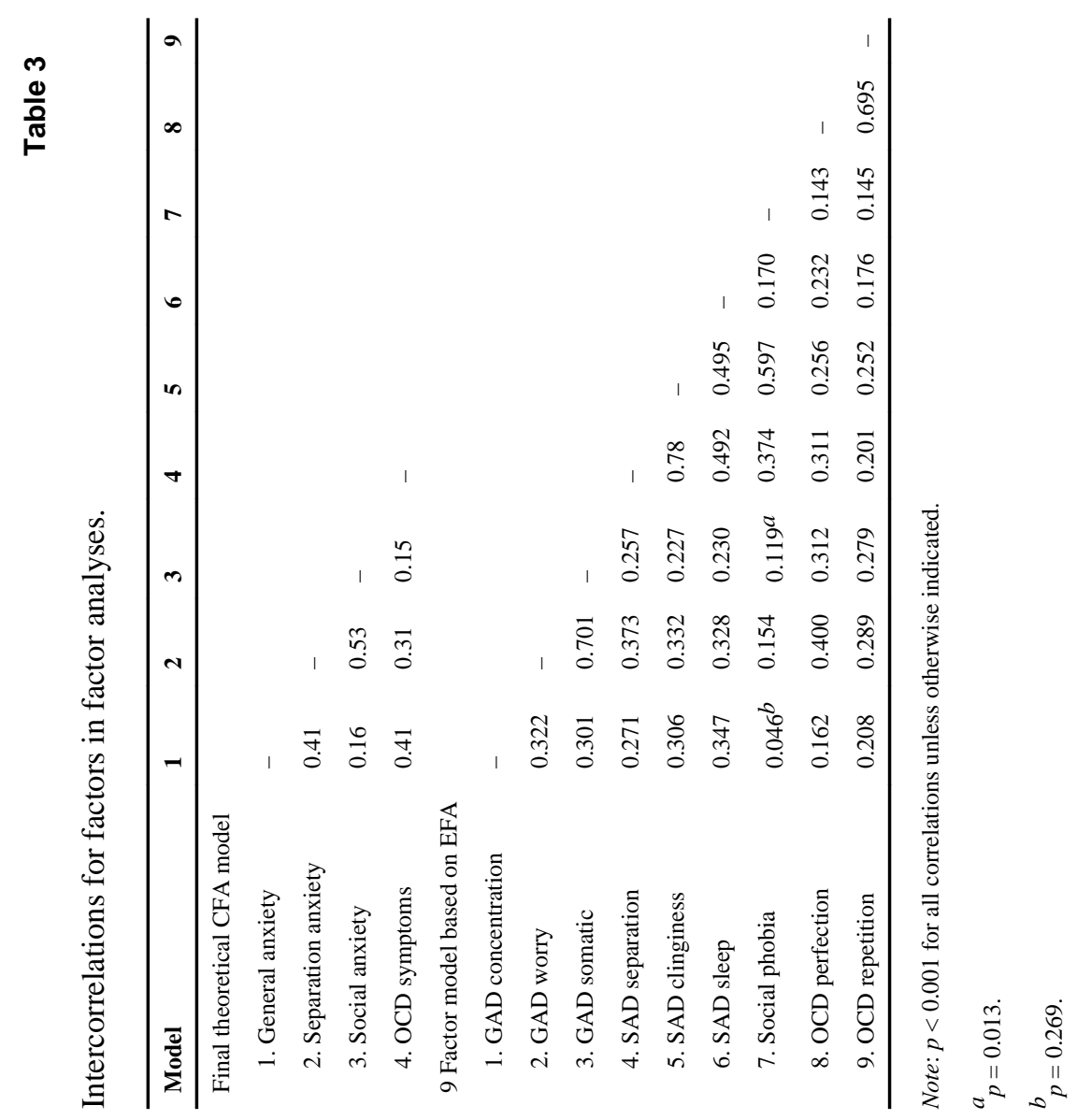

J Anxiety Disord. Author manuscript; available in PMC 2015 January 29. 


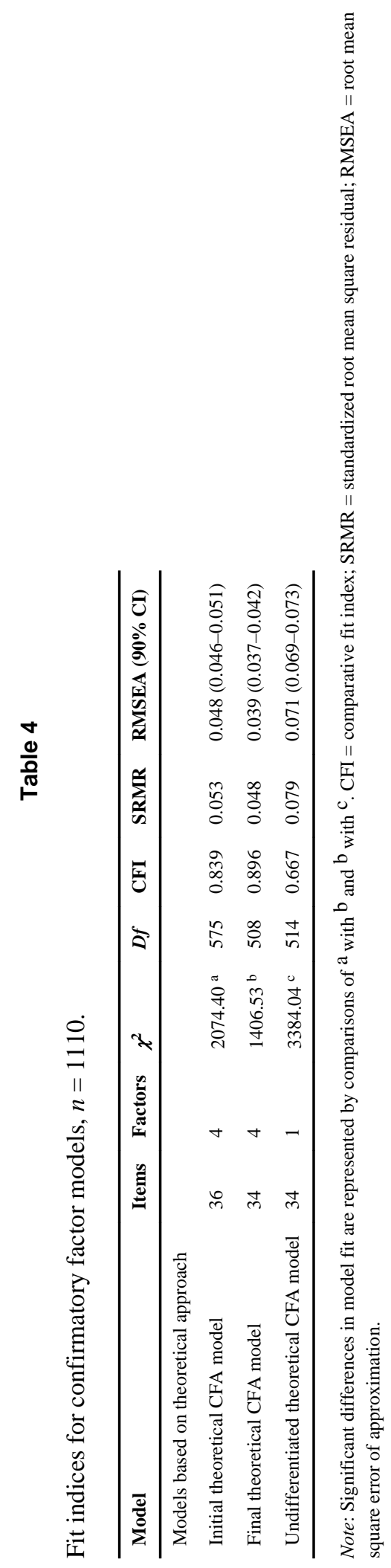

J Anxiety Disord. Author manuscript; available in PMC 2015 January 29. 